\title{
Determination of the electromagnetic character of soft dipole modes solely based on quasicontinuous $\gamma$ spectroscopy
}

\author{
A. Voinov* \\ Frank Laboratory of Neutron Physics, Joint Institute of Nuclear Research, 141980 Dubna, Moscow region, Russia \\ A. Schiller ${ }^{\dagger}$ \\ Lawrence Livermore National Laboratory, L-414, 7000 East Avenue, Livermore CA-94551 \\ M. Guttormsen, J. Rekstad, and S. Siem \\ Department of Physics, University of Oslo, N-0316 Oslo, Norway
}

\begin{abstract}
We show that the combined analysis of the quasicontinuous $\gamma$ spectra from the $\left({ }^{3} \mathrm{He}, \alpha\right)$ and the $\left(n_{\mathrm{th}}, 2 \gamma\right)$ reactions gives the possibility to measure the electromagnetic character of soft dipole resonances. Two-step $\gamma$-cascade spectra have been calculated, using level densities and radiative strength functions from the $\left({ }^{3} \mathrm{He}, \alpha \gamma\right)$ reaction. The calculations show that the intensity of the twostep cascades depends on the electromagnetic character of the soft dipole resonance under study. The difference reaches $40-100 \%$ which can be measured experimentally.
\end{abstract}

PACS number(s): 25.20.Lj, 24.30.Gd

\section{INTRODUCTION}

A soft resonance was observed for the first time by Jackson and Kinsey [1] in the $(n, \gamma)$ reaction for heavy nuclei $(A>70)$ near closed shells as an 'anomalous' bump appearing in the quasicontinuous $\gamma$ spectrum at $E_{\gamma} \sim 5.5 \mathrm{MeV}$. This resonance was called pygmy resonance $(\mathrm{PR})$ because of its small strength compared to the known, giant dipole resonance observed in photonuclear reactions [2]. Later, a similar 'anomalous' bump was observed for the well-deformed rare-earth nucleus ${ }^{170} \mathrm{Tm}$, but at the considerably lower energy of $E_{\gamma} \sim 3.0 \mathrm{MeV}$ [3]. A systematic investigation of the PR in the rare-earth mass region and beyond was made by Igashira et al. 沟, where they found that the centroid of the PR is approximately proportional to the mass number $A$ and varying from as low as $1.5 \mathrm{MeV}$ for ${ }^{142} \mathrm{Pr}$ to $5.6 \mathrm{MeV}$ for ${ }^{198} \mathrm{Au}$. While all those studies have been performed using radiative neutron capture, the PR was recently observed also in the radiative strength function (RSF) derived from the $\left({ }^{3} \mathrm{He}, \alpha \gamma\right)$ reaction [5]. This supports the assumption that the fundamental origin of this phenomenon is independent of the type of nuclear reaction.

However, in spite of the fact that the PR has been studied for a long time, the type of nuclear excitation responsible for the PR in the RSF remains unknown. Theory [6] predicts an $E 1 \mathrm{PR}$ around $5.5 \mathrm{MeV}$ in ${ }^{208} \mathrm{~Pb}$. The origin for the PR in this calculation is a supposed concentration of electric dipole strength in few low-energy states composed of $3 p^{-1} 4 s, 3 p^{-1} 3 d$, and $2 f^{-1} 3 d$ neutron configurations which are relatively weakly coupled to the giant electric dipole resonance (GEDR). This model suggest a quite strong $\mathrm{PR}$ which is not supported by experimental data. However, recently some indications in $\left(\gamma, \gamma^{\prime}\right)$ experiments for the existence of a weak $E 1 \mathrm{PR}$ in lead nuclei have been discussed [7]. On the other hand, the hypothesis has been suggested [8] that the PR observed at $E_{\gamma} \sim 3.0 \mathrm{MeV}$ in the quasicontinuous $\gamma$ spectra of well-deformed rare-earth nuclei and the so-called 'scissors mode' excitation studied by the nuclear resonance fluorescence method (NRF) [9] have the same physical origin. This would imply $M 1$ multipolarity for the PR and is supported by the similarity of the measured resonance parameters in the quasicontinuous $\gamma$ spectrum on the one hand, and the averaged properties of individual $M 1$ transitions seen in NRF experiments on the other hand. Finally, one can not exclude the possibility that the multipolarity of the PR can be different for different nuclei, i.e., one has to deal with different phenomena in different mass or energy regions. In conclusion, while the commonly-called pygmy resonance is most probably a dipole resonance, the electromagnetic character of this soft dipole mode remains unknown.

In NRF experiments, one can, in principle, measure the multipolarity and character of the involved $\gamma$ transitions, but one is usually limited to the investigation of the soft dipole resonances built on the ground state. Even so, for reliable results above $\sim 4 \mathrm{MeV}$, one should prefer the use of a polarized photon beam over common Compton polarimeters 10]. Anyway, in order to study average properties of the soft dipole modes built on excited states, one has to analyze quasicontinuous $\gamma$ spec-

*Electronic address: voinov@nf.jinr.ru

${ }^{\dagger}$ Electronic address: Andreas.Schiller@llnl.gov 
tra. To do so using a polarimeter might prove very hard, since the soft dipole mode is in general a weak resonance superimposed on the rather large tail of the GEDR [5] giving a very low signal-to-background ratio in the analysis. Unfortunately, with the exclusion of Compton polarimetry, there are no other known direct experimental methods ${ }^{1}$ to determine the electromagnetic character of soft dipole modes in quasicontinuous $\gamma$ spectroscopy. In this work, we propose the possibility to determine the character of soft dipole modes solely by using quasicontinuous $\gamma$ spectra from two different types of reactions, namely the $\left({ }^{3} \mathrm{He}, \alpha \gamma\right)$ and the $(n, 2 \gamma)$ reactions applied to the study of the same nucleus. This will enable us to study soft dipole modes built on excited states and their response to finite nuclear temperature.

\section{II. $\left({ }^{3} \mathrm{HE}, \alpha \gamma\right)$ REACTION}

The $\left({ }^{3} \mathrm{He}, \alpha \gamma\right)$ reaction is used for the extraction of level densities and RSFs from primary $\gamma$ spectra $P\left(E_{i}, E_{\gamma}\right)$ for excitation energies $E_{i}$ between 0 and the neutron binding energy $B_{n}$ and for spins between 2 and $6 \hbar$. The fundamental assumption behind the extraction procedure is the Brink-Axel hypothesis [11,12 which implies that the probability of radiative decay in the statistical regime, represented by the primary $\gamma$ spectra, can be expressed simply as a product of the final-state level density $\rho\left(E_{f}=E_{i}-E_{\gamma}\right)$ and the $\gamma$-ray transmission coefficient $T\left(E_{\gamma}\right)$

$$
P\left(E_{i}, E_{\gamma}\right) \propto T\left(E_{\gamma}\right) \rho\left(E_{f}\right) .
$$

The $\gamma$-ray transmission coefficient is now proportional to $\sum_{X L} E_{\gamma}^{2 L+1} f^{X L}\left(E_{\gamma}\right)$, where $f^{X L}\left(E_{\gamma}\right)$ is the RSF of the multipolarity $X L$. The details of the experimental extraction procedure have been described in Refs. [13,14] and references therein.

In Ref. [5], the total RSFs has been obtained from such experiments and analyzed for the ${ }^{161,162}$ Dy and ${ }^{171,172} \mathrm{Yb}$ nuclei. It has been established that the total RSF can be readily described by the sum of

- the tail of the GEDR as given by the KadmenskiuMarkushev-Furman (KMF) model [15] with a fixed nuclear temperature fitted to the experimental data

- the Lorentzian-shaped, single-humped spin-flip giant magnetic dipole resonance (GMDR) located around $7 \mathrm{MeV}$ of excitation energy

- a Lorentzian-shaped soft dipole mode or PR, where the resonance parameters were determined from experiment [5].

\footnotetext{
${ }^{1}$ The involved transition energies are in general too high for an efficient use of conversion electrons for multipolarity and character determination.
}

Although the centroid, width and strength of the soft dipole mode could be determined from experiment, its electromagnetic character could not. Thus, the question still remains open, whether it is of $E 1$ or $M 1$ type. The remaining of this work is therefore devoted to describing a method of determining the character of soft dipole modes observed in quasicontinuous $\gamma$ spectra.

\section{III. $(N, 2 \gamma)$ REACTION}

In the $(n, 2 \gamma)$ reaction with thermal neutrons, the compound state $i$ is formed with spin and parity $J_{i}^{\pi}=$ $I^{\pi} \pm 1 / 2$, where $I^{\pi}$ is the spin and parity of the target nucleus. The most intense two-step $\gamma$ cascades (TSCs) accessible to experimental study populate low-lying excited states with excitation energies $E_{f}$ up to around $1 \mathrm{MeV}$ 16.

Thus, TSCs start from the initial (compound) state $i$ with a certain spin and parity to a final state $f$ through all possible intermediate levels $j$ with excitation energies from $E_{f}$ to $E_{i}=B_{n}$. The possible spins of the intermediate levels $J_{j}^{\pi}$ are determined by the multipolarities in the TSCs. Assuming that the most intense TSCs have dipole multipolarity, the most probable spin interval for the intermediate level can be defined as

$$
\max \left[J_{i}-1, J_{f}-1\right] \leq J_{j} \leq \min \left[J_{i}+1, J_{f}+1\right]
$$

Assuming the validity of the statistical theory, the intensity of TSCs between $i \rightarrow j \rightarrow f$ levels is determined by the partial and total radiative widths of both, the initial $\Gamma_{i j}, \Gamma_{i}$ and intermediate $\Gamma_{j f}, \Gamma_{j}$ levels, respectively

$$
I_{i j f}^{\gamma \gamma}=\frac{\Gamma_{i j}}{\Gamma_{i}} \frac{\Gamma_{j f}}{\Gamma_{j}} .
$$

Partial radiative widths of any arbitrary level $l$ can be expressed in terms of the $\operatorname{RSF} f^{X L}\left(E_{\gamma}\right)$

$$
\Gamma_{l m}^{X L}\left(E_{\gamma}=E_{l}-E_{m}\right)=f^{X L}\left(E_{\gamma}\right) E_{\gamma}^{(2 L+1)} D_{l}\left(E_{l}\right),
$$

where $D_{l}$ is the energy spacing of levels having the same spin and parity as $l$ [17]. The total radiative width is determined by the sum of all partial radiative widths $\Gamma_{l m}$ summed over all $\gamma$ transitions populating levels $m$ lying below $l$ in excitation energy, i.e., $\Gamma_{l}=\sum_{m<l} \Gamma_{l m}$.

The multipolarities of the transitions in a TSC depend on the spins and parities of the connected states. Here, we assume that the main contributions come from $E 1$ and $M 1 \gamma$ transitions. Also some E2 strength will be 
present, but is neglected in the present discussion. Thus, if the parities of the initial $i$ and final $f$ states are different, the $(E 1, M 1)$ and $(M 1, E 1)$ sequences of transition multipolarities are possible. In the case of equal parities of the initial $i$ and final $f$ states, the $\gamma$-transitions of the TSCs have $(E 1, E 1)$ and $(M 1, M 1)$ multipolarities. Because there is in general no possibility to distinguish between primary $E_{\gamma_{1}}$ and secondary $E_{\gamma_{2}}$ transitions in the cascade, ${ }^{2}$ the observed spectrum with the total TSC energy $E_{\text {tot }}=E_{\gamma_{1}}+E_{\gamma_{2}}=B_{n}-E_{f}$ is symmetric with respect to the energy $E_{\gamma}=E_{\text {tot }} / 2$ and is determined by

$$
\begin{aligned}
& \frac{\mathrm{d} N\left(E_{\gamma} ; E_{\mathrm{tot}}\right)}{\mathrm{d} E_{\gamma}}= \\
& \sum_{\substack{L, L^{\prime}, J \\
X, X^{\prime}, \pi}} \frac{\Gamma_{i j}^{X L}\left(E_{\gamma}\right)}{\Gamma_{i}} \frac{\Gamma_{j f}^{X^{\prime} L^{\prime}}\left(E_{\mathrm{tot}}-E_{\gamma}\right)}{\Gamma_{j}} \rho_{j}^{J^{\pi}}\left(B_{n}-E_{\gamma}\right) \\
& +\sum_{\substack{L, L^{\prime}, J \\
X, X^{\prime}, \pi}} \frac{\Gamma_{i h}^{X L}\left(E_{\mathrm{tot}}-E_{\gamma}\right)}{\Gamma_{i}} \frac{\Gamma_{h f}^{X^{\prime} L^{\prime}}\left(E_{\gamma}\right)}{\Gamma_{h}} \rho_{h}^{J^{\pi}}\left(E_{f}+E_{\gamma}\right)
\end{aligned}
$$

where $\rho_{j}$ and $\rho_{h}$ denote the density of intermediate levels populated by TSCs, and the sums run over all possible combinations of $L, L^{\prime}, J$ and $X, X^{\prime}, \pi$. Thus, we notice that the quasicontinuous $\gamma$-spectra from both, the $\left({ }^{3} \mathrm{He}, \alpha \gamma\right)$ and the $(n, 2 \gamma)$ reaction are determined by the same level densities and RSFs. This allows us to use the quantities which can be extracted from the $\left({ }^{3} \mathrm{He}, \alpha \gamma\right)$ reaction in order to calculate the quasicontinuous spectra of TSCs following thermal neutron capture. In the following, we will mainly focus on the influence of the unknown electromagnetic character of the soft dipole mode on calculated TSC spectra.

\section{CHARACTER OF SOFT DIPOLE MODES}

For the sake of simplicity, we first consider the TSC with the $(E 1, M 1)$ sequence of transition multipolarities. If the soft dipole mode is of $M 1$ character, the corresponding strength function $f^{\mathrm{SD}}$ should be added incoherently to the spin-flip GMDR strength function $f^{\mathrm{SF}}$ giving the total magnetic dipole strength function $f^{M 1}=f^{\mathrm{SF}}+f^{\mathrm{SD}}$. The same is correct for the radiative widths $\Gamma^{M 1}=\Gamma^{\mathrm{SF}}+\Gamma^{\mathrm{SD}}$. The $E 1$ strength function is then completely determined by the tail of the GEDR (modeled in this work by the KMF approach). The intensity of $(E 1, M 1)$ TSCs can therefore be written as

$$
I_{i j f}^{\mathrm{SD}=M 1}=\frac{\Gamma_{i j}^{\mathrm{KMF}}\left(E_{\gamma_{1}}\right)}{\Gamma_{i}} \frac{\left[\Gamma_{j f}^{\mathrm{SF}}\left(E_{\gamma_{2}}\right)+\Gamma_{j f}^{\mathrm{SD}}\left(E_{\gamma_{2}}\right)\right]}{\Gamma_{j}}
$$

or

$$
I_{i j f}^{\mathrm{SD}=M 1}=\frac{\Gamma_{i j}^{\mathrm{KMF}}\left(E_{\gamma_{1}}\right)}{\Gamma_{i}} \frac{\Gamma_{j f}^{\mathrm{SF}}\left(E_{\gamma_{2}}\right)}{\Gamma_{j}}\left[1+\frac{\Gamma_{j f}^{\mathrm{SD}}\left(E_{\gamma_{2}}\right)}{\Gamma_{j f}^{\mathrm{SF}}\left(E_{\gamma_{2}}\right)}\right] .
$$

In the case of the soft dipole mode being of $E 1$ character, the total electric dipole strength function $f^{E 1}$ is given by the incoherent sum of the KMF strength function $f^{\mathrm{KMF}}$ and the soft dipole resonance $f^{\mathrm{SD}}$, i.e., $f^{E 1}=f^{\mathrm{KMF}}+f^{\mathrm{SD}}$. The intensity of the $(E 1, M 1)$ cascade can then be expressed as

$$
I_{i j f}^{\mathrm{SD}=E 1}=\frac{\Gamma_{i j}^{\mathrm{KMF}}\left(E_{\gamma_{1}}\right)}{\Gamma_{i}} \frac{\Gamma_{j f}^{\mathrm{SF}}\left(E_{\gamma_{2}}\right)}{\Gamma_{j}}\left[1+\frac{\Gamma_{i j}^{\mathrm{SD}}\left(E_{\gamma_{1}}\right)}{\Gamma_{i j}^{\mathrm{KMF}}\left(E_{\gamma_{1}}\right)}\right] .
$$

The ratio of the cascade intensities (7) and (8) is finally determined by

$$
\frac{I_{i j f}^{\mathrm{SD}=E 1}}{I_{i j f}^{\mathrm{SD}}=M 1}=\frac{\left[1+\frac{\Gamma_{i j}^{\mathrm{SD}}\left(E_{\gamma_{1}}\right)}{\Gamma_{i j}^{\mathrm{KMF}}\left(E_{\gamma_{1}}\right)}\right]}{\left[1+\frac{\Gamma_{j f}^{\mathrm{SD}}\left(E_{\gamma_{2}}\right)}{\Gamma_{j f}^{\mathrm{SF}}\left(E_{\gamma_{2}}\right)}\right]} .
$$

One can see that this ratio depends on the ratios of the soft dipole and the KMF-GEDR strength function and on the ratio of the soft dipole and the spin-flip GMDR strength function for primary $\left(i \rightarrow j, E_{\gamma_{1}}\right)$ and secondary $\left(j \rightarrow f, E_{\gamma_{2}}\right)$ transitions, respectively. It is known from Ref. [5] that the spin-flip GMDR strength function $f^{\mathrm{SF}}$ is less than $\sim 20 \%$ in magnitude than the tail of the GEDR strength function $f^{\mathrm{KMF}}$ according to the KMF model. Therefore, it is expected that the intensity of the calculated TSCs will be higher in the case of $M 1$ character of the soft dipole resonance under study than for $E 1$ character, provided that the parities of the initial and final state are opposite. The main differences in the calculated TSC spectra for the two possible characters of the soft dipole mode are expected to be located around its centroid, i.e., in the region of $E_{\gamma} \sim 2.5-3.5 \mathrm{MeV}$ for deformed rare earth nuclei.

By analogy with Eqs. (7) and (8), similar expressions can be written for the $(M 1, E 1)$ sequence of multipolarities in the TSC. For the $(E 1, E 1)$ and the $(M 1, M 1)$ sequences, i.e., for equal parities of the initial and final state, the situation is more complex and it is more difficult to draw conclusions from simple relations similar to Eqs. (6/9). In general, one can state that in these cases, the calculated TSC intensities will be higher for $E 1$ than for $M 1$ multipolarity of the soft dipole mode

\footnotetext{
${ }^{2}$ Ordering the $\gamma$ transitions in TSC experiments has been attempted in, e.g., 18.
} 
under study, but the differences between the two calculations are smaller than in the case of opposite parities of the initial and final state.

In order to investigate the intensity difference of calculated TSC spectra due to the two possible characters of the soft dipole resonance, we have calculated such spectra according to Eq. (5) for the two even-even nuclei ${ }^{162} \mathrm{Dy}$ and ${ }^{172} \mathrm{Yb}$ and the odd nucleus ${ }^{161} \mathrm{Dy}$. For all of these nuclei, the level density and total RSF, as well as the resonance parameters of the soft dipole mode have been obtained from $\left({ }^{3} \mathrm{He}, \alpha \gamma\right)$ experiments [5]. We will investigate TSCs populating the first $2^{+}$level of the ${ }^{162} \mathrm{Dy}$ and ${ }^{172} \mathrm{Yb}$ nuclei. The initial states (which are formed at $B_{n}$ ) have mainly spin and parity $J_{i}^{\pi}=3^{+}$and $J_{i}^{\pi}=0^{-}$ for the ${ }^{162} \mathrm{Dy}$ and ${ }^{172} \mathrm{Yb}$ nuclei, respectively. Thus, we can investigate the $(E 1, E 1)$ and $(M 1, M 1)$ sequences of TSCs in the case of the ${ }^{162}$ Dy nucleus and the $(E 1, M 1)$ and $(M 1, E 1)$ sequences in the case of the ${ }^{172} \mathrm{Yb}$ nucleus. The ${ }^{161}$ Dy nucleus is interesting because it has two low lying levels $5 / 2^{+}$and $5 / 2^{-}$which are very close in excitation energy (0 and $25.6 \mathrm{keV}$ respectively) and which have the same spin but opposite parity. The initial state (formed by thermal neutron capture) has $J_{i}^{\pi}=1 / 2^{+}$. Thus, we can investigate $(E 1, E 1)$ and $(M 1, M 1)$ as well as $(E 1, M 1)$ and $(M 1, E 1)$ cascades for the same nucleus.

Because the spin interval accessible for $\gamma$-transitions is different for the $\left({ }^{3} \mathrm{He}, \alpha \gamma\right)$ and $(n, 2 \gamma)$ reaction, a spin distribution of the level density has to be taken into account. We assume the spin distribution according to [19]

$$
g(E, I)=\frac{2 I+1}{2 \sigma^{2}} \exp \left[-(I+1 / 2)^{2} / 2 \sigma^{2}\right],
$$

where $\sigma$ is the excitation-energy dependent spin cut-off parameter. Furthermore, we assume equal amounts of levels with positive and negative parities for all relevant spins and excitation energies.

The calculated TSC spectra for the different nuclei and final states are given in Figs. 11 and 2. The calculations were carried out for both possible characters of the soft dipole mode, giving two solutions. The summed intensities of all TSCs (the integrals over the TSC spectra) populating a fixed low lying level are shown in Table 1 . The results of the calculations show that the two possible electromagnetic characters of the soft dipole resonance yield different spectral distributions of TSCs as well as different summed intensities. In the more favorable cases where the parities of the initial and final states are opposite, the summed intensity differs between $30 \%$ for ${ }^{172} \mathrm{Yb}$ and up to a factor of two for ${ }^{161} \mathrm{Dy}$ with the $M 1$ multipolarity for the soft dipole mode resulting in the higher intensities. The spectral distributions give differences of up to a factor of two to three at energies which correspond to the centroid of the soft dipole resonance and show markedly different shapes. For the less favorable cases where the parities of the initial and final states are equal, the differences in summed intensities amounts only to $15 \%$ for the ${ }^{162}$ Dy nucleus and $\sim 45 \%$ for the ${ }^{161}$ Dy nucleus with the $E 1$ multipolarity giving the higher intensi- ties. The spectral distributions for the two possible electromagnetic characters differ between $25 \%$ and a factor of two around the centroid of the soft dipole resonance. Again the shapes of the TSCs spectra show differences as well.

Thus, we see that the intensity of the TSC transitions can depend strongly on the character of the soft dipole resonance under study. The effect should certainly be large enough to be measured experimentally, especially for TSCs with opposite parities of the initial and final states connected by the TSC transitions. This fact should open the possibility to use the experimental measurements of TSC spectra to determinate the character of soft dipole modes observed in the analysis of $\left({ }^{3} \mathrm{He}, \alpha \gamma\right)$ reaction data, i.e., using solely quasicontinuous $\gamma$ spectroscopy.

\section{POSSIBLE SYSTEMATIC ERRORS AND OTHER CONSIDERATIONS}

It should be noted that the statements and conclusions discussed above are based on some fundamental assumptions. The most important of these is that the $\gamma$ emission in both reactions discussed is of statistical nature. For the $\left({ }^{3} \mathrm{He}, \alpha \gamma\right)$ reaction, this means that the decay properties of the ensemble of reaction selected states within a certain energy bin are independent of whether these states are directly populated by $\alpha$ particles through the nuclear reaction or by $\gamma$-transitions from higher excited states. This is believed to be approximately fulfilled because the relatively long life time of the excited states $\left(\sim 10^{-15} \mathrm{~s}\right)$ gives the nucleus time to thermalize prior to radiative decay. The same is assumed for the $(n, 2 \gamma)$ reaction, i.e., it is assumed that the reaction proceeds through a compound state which has a long life time and that the radiative decay properties of this compound state are independent of its formation. It is also assumed for both reactions that the mean values of the radiative widths $\left\langle\Gamma_{i f}\right\rangle$ are independent on the structure of the connected states and are defined by the relevant level spacings and RSFs.

These assumptions have been partly tested for the ${ }^{162}$ Dy nucleus 5. It has been shown that the measured total $\gamma$-ray spectrum of the ${ }^{161} \operatorname{Dy}(n, \gamma){ }^{162}$ Dy reaction with $\mathrm{keV}$ neutrons can be very well reproduced by using the level density and RSFs obtained from the ${ }^{163} \mathrm{Dy}\left({ }^{3} \mathrm{He}, \alpha \gamma\right){ }^{162} \mathrm{Dy}$ reaction. Additionally, for the ${ }^{162}$ Dy nucleus, the essentially same level density and RSF have been obtained from the ${ }^{162} \mathrm{Dy}\left({ }^{3} \mathrm{He},{ }^{3} \mathrm{He} \gamma\right){ }^{162} \mathrm{Dy}$ reaction 20]. These results strongly support the statistical model for the radiative decay in these reactions and the correctness of the extraction procedure for level densities and RSFs in the case of the ${ }^{3} \mathrm{He}$ induced reactions.

A second difficulty in the above outlined method to measure the multipolarity of soft dipole resonances comes from the fact that the extraction procedure for level densities and RSFs applied to the $\left({ }^{3} \mathrm{He}, \alpha \gamma\right)$ reaction allows 
us to get only the total RSF, i.e., the incoherent sum of all multipolarities. Decomposition of the total RSF into strength functions connected to definite multipolarities is determined by corresponding models used to describe the $E 1$ and $M 1$ strength in nuclei. This model dependent analysis can, in principle, give additional uncertainties to the analysis of TSC spectra. However, it has been shown [5] that if one tests only widely accepted models of E1 and $M 1$ strength functions in well-deformed rare earth nuclei, the total RSF extracted from the $\left({ }^{3} \mathrm{He}, \alpha \gamma\right)$ reaction allows one to select only the KMF model with a constant nuclear temperature for the tail of the GEDR, a single-humped Lorentzian for the spin-flip GMDR resonance, ${ }^{3}$ and a Lorentzian for the soft dipole mode. From this point of view, the measurements of TSC spectra can also be considered as an additional test of this decomposition.

Finally, investigating the same residual nucleus by the $\left({ }^{3} \mathrm{He}, \alpha \gamma\right)$ and $(n, 2 \gamma)$ reaction, requires two target nuclei with neutron number differing by two units. If we restrict the search to stable targets of well-deformed rare-earth nuclei, only 22 of such pairs of target nuclei exist between Gd and Hf. ${ }^{4}$ Unfortunately, until now, there is, to our knowledge, no published data set from both reactions populating the same residual nucleus. Thus, the analysis outlined in this work can not be performed without additional experimental efforts.

\section{CONCLUSION}

We have shown that a joint experimental investigation of the same product nucleus by the two different reactions $\left({ }^{3} \mathrm{He}, \alpha \gamma\right)$ and $(n, 2 \gamma)$, can be used to determine the character of soft dipole resonances observed in the RSF. The first reaction is used for extracting the level density and the total RSF of the residual nucleus. The subsequent decomposition of this total RSF into the tail of the GEDR, the GMDR, and the soft dipole resonance is model dependent and subject to possible systematic errors. The second reaction can then be used to determine the character of the soft dipole resonance via the measurement of TSC intensities. The most suitable TSCs for this analysis are those with opposite parity of the initial and final level. The results from the $(n, 2 \gamma)$ experiment can also be used as an additional test for the correct decomposition of the total RSF obtained from the $\left({ }^{3} \mathrm{He}, \alpha \gamma\right)$ experiment into modeled electric and magnetic

\footnotetext{
${ }^{3}$ Double-humped Lorentzians as proposed in 21] were not considered in the decomposition.

${ }^{4}$ The method outlined in this work should be applicable to any nucleus. However, most of the published $\left({ }^{3} \mathrm{He}, \alpha \gamma\right)$ and $(n, 2 \gamma)$ experiments have been devoted to well-deformed rareearth nuclei.
}

dipole strength functions. Finally, the exclusive use of quasicontinuous $\gamma$ spectra for determining the character of soft dipole modes gives us, in contrast to typical NRF experiments, for the first time the opportunity to study soft dipole resonances built on excited states and their response to finite nuclear temperatures.

\section{ACKNOWLEDGMENTS}

Part of this work was performed under the auspices of the U.S. Department of Energy by the University of California, Lawrence Livermore National Laboratory under Contract No. W-7405-ENG-48. Financial support from the Norwegian Research Council (NFR) is gratefully acknowledged. A.V. would like to thank the staff of the University of Oslo for their warm hospitality while this manuscript was prepared and acknowledges support from a NATO Science Fellowship under project number 150027/432 given through the Norwegian Research Council (NFR).

[1] J. David Jackson and B. B. Kinsey, Minutes of the 1951 Annual Meeting Held at New York, February 1-3, 1951, Phys. Rev. 82, 345 (1951).

[2] Samuel S. Dietrich and Barry L. Berman, At. Data Nucl. Data Tables 38, 199 (1988).

[3] S. Joly, D. M. Drake, and L. Nilsson, Phys. Rev. C 20, 2072 (1979).

[4] M. Igashira, H. Kitazawa, M. Shimizu, H. Komano, and N. Yamamuro, Nucl. Phys. A457, 301 (1986).

[5] A. Voinov, M. Guttormsen, E. Melby, J. Rekstad, A. Schiller, and S. Siem, Phys. Rev. C 63, 044313 (2001).

[6] A. M. Lane, Ann. Phys. (N.Y.) 63, 171 (1971).

[7] J. Enders et al., Phys. Lett. B 486, 279 (2000).

[8] A. Schiller, M. Guttormsen, E. Melby, J. Rekstad, S. Siem, and A. Voinov, preprint nucl-ex/0011018.

[9] N. Pietralla, P. von Brentano, R.-D. Herzberg, U. Kneissl, N. Lo Iudice, H. Maser, H. H. Pitz, and A. Zilges, Phys. Rev. C 58, 184 (1998), and references therein.

[10] N. Pietralla et al., Phys. Rev. Lett. 88, 012502 (2002).

[11] D. M. Brink, Ph.D. thesis, Oxford University, 1955.

[12] P. Axel, Phys. Rev. 126, 671 (1962).

[13] L. Henden, L. Bergholt, M. Guttormsen, J. Rekstad, and T. S. Tveter, Nucl. Phys. A589, 249 (1995). 
[14] A. Schiller, L. Bergholt, M. Guttormsen, E. Melby, J. Rekstad, and S. Siem, Nucl. Instrum. Methods Phys. Res. A 447, 498 (2000).

[15] S. G. Kadmenskiu, V. P. Markushev, and V. I. Furman, Yad. Fiz. 37, 277 (1983) [Sov. J. Nucl. Phys. 37, 165 (1983)].

[16] E. V. Vasil'eva, A. M. Sukhovoi, V. A. Khitrov, Fiz. Elem. Chastits Atom. Yadra 31, 350 (2000) [Phys. Part. Nucl. 31, 170 (2000)].

[17] G. A. Bartholomew, E. D. Earle, A. J. Ferguson, J.
W. Knowles, and M. A. Lone, Adv. Nucl. Phys. 7, 229 (1973).

[18] M. A. Ali, V. A. Khitrov, Yu. V. Kholnov, A. M. Sukhovoj, and A. V. Voinov, J. Phys. G 20, 1943 (1994).

[19] A. Gilbert and A. G. W. Cameron, Can. J. Phys. 43, 1446 (1965).

[20] A. Schiller, M. Guttormsen, E. Melby, J. Rekstad, and S. Siem, Phys. Rev. C 61, 044324 (2000).

[21] A. Richter, Prog. Part. Nucl. Phys. 34, 261 (1995). 
TABLE I. Summed TSC intensities $\sum_{j} I_{i j f}^{\gamma \gamma}$ over all intermediate states $j$, and calculated under the assumption of $E 1$ or $M 1$ multipolarity of the soft dipole resonance.

\begin{tabular}{cccccccc}
\hline \hline nucleus & \multicolumn{2}{c}{${ }^{162} \mathrm{Dy}$} & \multicolumn{2}{c}{${ }^{172} \mathrm{Yb}$} & \multicolumn{3}{c}{${ }^{161} \mathrm{Dy}$} \\
$J_{i}^{\pi} \rightarrow J_{f}^{\pi}$ & \multicolumn{2}{c}{$3^{+} \rightarrow 2^{+}$} & \multicolumn{2}{c}{$0^{-} \rightarrow 2^{+}$} & \multicolumn{2}{c}{$1 / 2^{+} \rightarrow 5 / 2^{+}$} & $M 1 / 2^{+} \rightarrow 5 / 2^{-}$ \\
multipolarity & $E 1$ & $M 1$ & $E 1$ & $M 1$ & $E 1$ & $E 1$ & $M 1$ \\
$\sum_{j} I_{i j f}^{\gamma \gamma}[\%$ per capture $]$ & 4.9 & 4.2 & 8.1 & 11.3 & 4.2 & 2.7 & 0.9 \\
\hline \hline
\end{tabular}




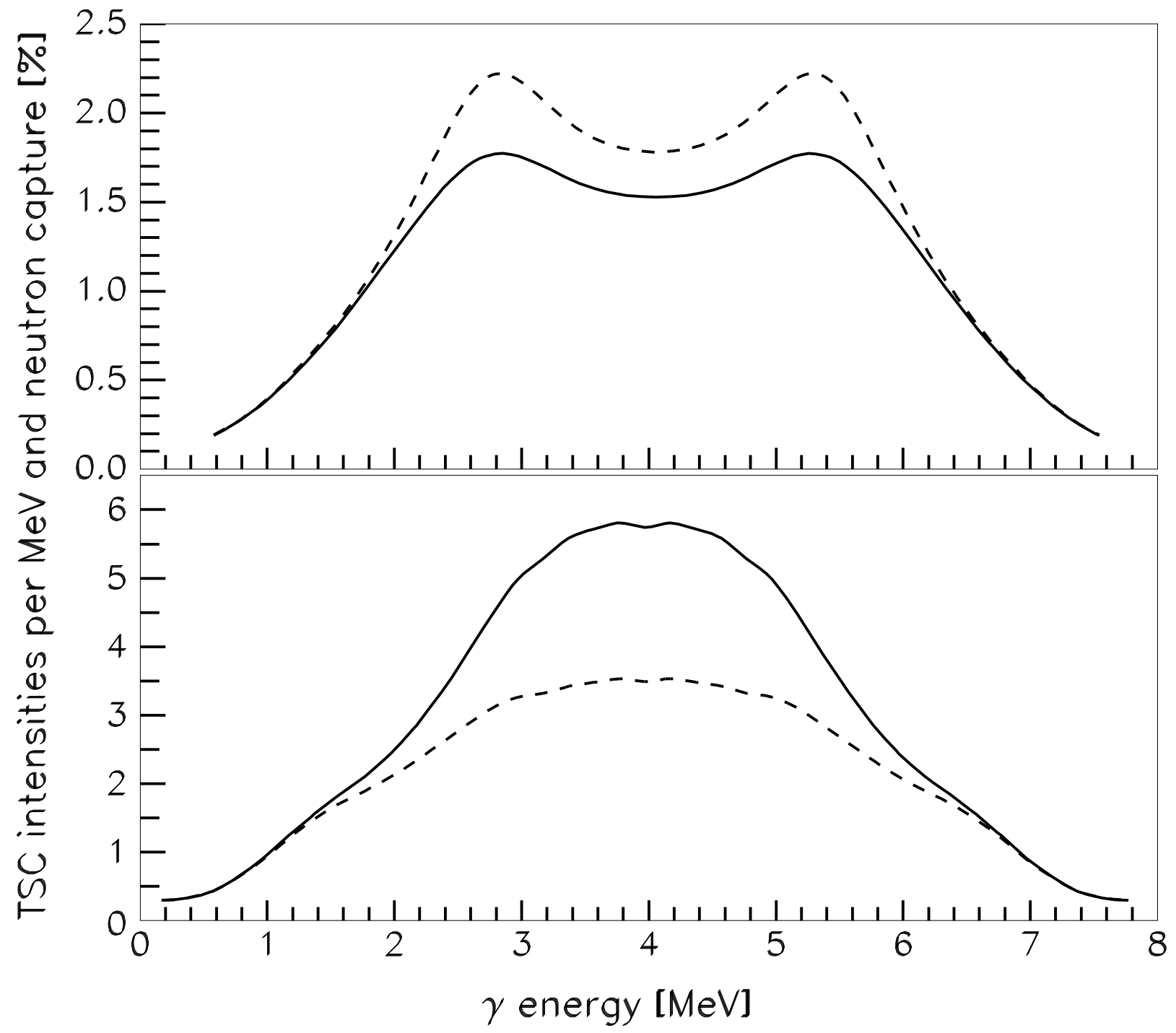

FIG. 1. Calculated spectra of TSCs to the first $2^{+}$state from the ${ }^{161} \operatorname{Dy}(n, 2 \gamma){ }^{162} \mathrm{Dy}$ (upper panel) and ${ }^{171} \mathrm{Yb}(n, 2 \gamma){ }^{172} \mathrm{Yb}$ (lower panel) reactions. Due to the unknown multipolarity of the soft dipole mode, we obtain two different solutions corresponding to $E 1$ (dashed line) or $M 1$ (solid line) multipolarity. Comparison to experimental data will reveal which of the $E 1$ or M1 assignment is the most probable. 




FIG. 2. Same as Fig. 1 1 but for the ${ }^{160} \mathrm{Dy}(n, 2 \gamma){ }^{161}$ Dy reaction and for two different final states with spins $5 / 2^{+}($upper panel) and $5 / 2^{-}$(lower panel). The calculations must describe experimental TSC spectra to both final spins simultaneously assuming one and the same multipolarity for the soft dipole mode. 